\title{
Model-driven Platform for Oil and Gas Enterprise Data Integration
}

\author{
Vadim Veyber \\ National Research Tomsk \\ Polytechnic University, \\ Tomsk, Russia
}

\author{
Anton Kudinov \\ National Research Tomsk \\ Polytechnic University, \\ Tomsk, Russia
}

\author{
Nikolay Markov \\ JSC VostokGazprom \\ Tomsk, Russia
}

\begin{abstract}
The necessity of data interoperability is very important for the oil and gas enterprise environment. Different information systems and applications cover the range of the IT infrastructure and need to interoperate. Most of the industrial companies do not have common rational strategy for their automation. That leads to the lack of integration between purchased and implemented software that is necessary for various departments. Integration problems prevent companies from building common information space. An instrumental platform for oil and gas enterprise data integration has been considered as the problem solution. The proposed platform combines Service-Oriented Architecture principles and Model-Driven Approach and uses branch standard PRODML as a model for object mapping.
\end{abstract}

\section{General Terms}

Model Driven Approach; Enterprise Applications Integration; Service Oriented Architecture; Integration using Web Services.

\section{Keywords}

Model Driven Approach; Enterprise Applications Integration; Service Oriented Architecture.

\section{INTRODUCTION}

Flows of information repeatedly increasing in the process of development of a large industrial enterprise and the proper organization of these flows within an enterprise becomes more important. Analysis of the problems of automation of oil and gas enterprises shows that the "patchwork automation" is characteristic to many of them. This can be explained by the fact that no one integrated information system, even on scale of huge ERP (Enterprise Resource Planning), does not cover all needs in automation of enterprise, especially its special requirements associated with the features of the production of continuous type. Today oil and gas enterprise automation provided by a variety of software, as a rule this automation is limited to only certain areas and production departments, but not the production cycle of an enterprise as a whole. All this points to the fact that problem of oil and gas enterprise information systems integration and especially the problem of common information space development of such enterprise are very important.

\section{PROBLEMS OF OIL AND GAS ENTERPRISE PRODUCTION DATA INTEGRATION}

\subsection{CIM model}

Computer Integrated Manufacturing (CIM) [1] is a wellestablished model of enterprise informatization satisfying the modern level of automation. According to this model, toplevel systems operate aggregate data in long-time intervals, and low-level systems operate a large flow of real-time data. To connect top-level systems data to information about realtime events controlled by low-level systems, intermediate Manufacturing Execution Systems (MES) are used. The lower layer of the model is represented by data gathering units (sensors), programmable devices (e.g. comptrollers for numerically controlled machine tools), automated supervisory control system SCADA (Supervisory for Control And Data Acquisition) and APCS (Automated Process Control System) which interact with the equipment directly.

APCS interact with MES by gathering technological processes data and allow controlling enterprise production processes efficiently. At the same time MES provides the aggregated information for such enterprise systems as ERP and BI (Business Intelligence) -systems for analytics. In practice, such huge and expensive multipurpose integration platforms as Microsoft BizTalk [2], IBM WebSphere [3], etc. are often used to integrate different levels of the enterprise. But using specialized integration solution for a certain domain can be much more effective in terms of both productivity and cost than the multipurpose solutions.

There are various common classifications of the integration types [4]. This work focuses on the integration type "by data" for information systems and industrial applications integration for oil and gas enterprise automation.

\subsection{Oil and gas enterprise data cycle}

According to CIM model, the interaction of enterprise information system has two directions of its implementation "horizontal" and "vertical". The "vertical" integration provides data exchange: (1) between the level of technological processes (APCS) and the level of factory floor production management (MES); (2) between MES-level and enterprise control level (ERP, BI). The "horizontal" integration provides data gathering within one level, for example, integration of MES and specialized information systems for oil and gas industry (for processing geological prospecting data, for the oil reservoirs modeling, etc.). 


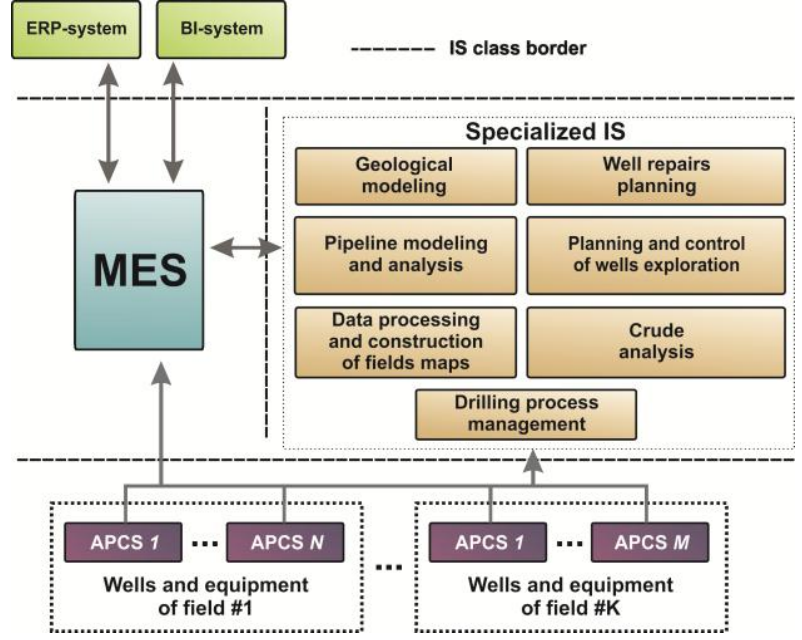

Fig. 1: Oil and gas enterprise information systems interaction

Let's consider the process of manufacturing data exchange in detail (fig. 1). Manufacturing data is gathered on oil and gas fields by means of APCS while processes of hydrocarbon crude materials producing, preparing and transporting. The considerable part of the data from the technological control level is delivered to the MES-level. The MES, first of all, automates the business processes of the industrial departments within different levels of production management; it allows planning and controlling technological activities for equipment maintenance and repair, coordinating functions of all the industrial departments within the enterprise. Some kinds of information (well condition data, volumes of extraction and preparation of hydrocarbon crude materials, the results of chemical analyses of production transferred in the main oil and gas pipelines) come into the MES as input data. Vice versa, some part of the data from the MES (the aggregated technological data, the data about well condition, etc.) is necessary for the information systems solving specialized data processing an analysis tasks. These tasks for the most of MES are beyond their functionality (for example, pipelines modeling, geological field models development, etc.). The part of data from MES which is usually aggregated and consolidated (production volume of hydrocarbon crude material, its leakage, its usage for technological purposes, etc.) should arrive into the ERP and BI systems i.e. on the higher level of enterprise management [5].

The process of oil and gas enterprise data integration is connected with a number of problems which significantly complicate both "vertical" and "horizontal" integration.

Firstly, the complexity of systems "horizontal" interaction can be explained by systems territorial distribution and heterogeneity of data transfer protocols. While developing, the enterprise implements stand-alone information systems and technological databases (DB) which creates a complicated information structure of the industrial data collecting and processing.

Secondly, at each enterprise the own set of specialized software and applications is formed. For example, as oil reservoir modeling tool - Eclipse by Schlumberger Company [7], as production control tool - Prosper and Gap [8] by Petroleum Experts and as a visualization system - ProcessNet by Matrikon [9] can co-exist within one enterprise. Problems | are_caused by fragmentary choice of the systems. The integration of the products, interaction of which is not provided by their developers, is an expensive and laborconsuming task required a lot of resources. On the other hand, total replacement of the existing systems is impossible due to the previous huge investments.

Thirdly, at any modern oil and gas enterprise there are great volumes of technological data (the information from sensors of industrial equipment, the results of chemical analyses, equipment maintenance and repair data, the geological data, etc.). These data volumes complicate the process of their transmission while integrating information systems and applications.

All the above-mentioned claims that solving problems of data integration for oil and gas enterprise is a rather labor intensive process.

\subsection{Oil and gas standards for integration}

Standardization is very important for manufacturing. Solving the problem of oil and gas data integration we need to focus on modern and commonly accepted standards and technologies used in chemical, petrochemical, oil and gas industries, which are directly or indirectly related to the problem of data integration. After analyzing the existing standards (fig. 2) we can conclude that there are no highly unified standards of integration in oil and gas branch for the above-mentioned tasks, the exception is standard OPC (OLE for Process Control) [6]. Although, OPC is not a specialized standard for the considered application domain, it perfectly solves the task of integration between APCS and MES.

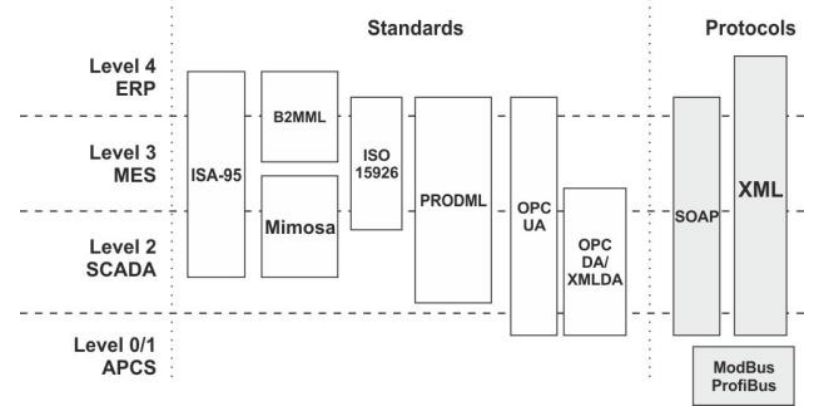

Fig. 2: Standards

On the other hand, analysis of PRODML standard showed that it is also useful for solving the problems of oil and gas data integration. Other considered integration standards are very abstract and appropriate only for the enterprises with the discrete production cycle.

\section{METAMODEL APPROACH TO DATA INTEGRATION}

Each information system realizes an original interpretation of application domain. Thus, the problem of data correlation is rather complex. The availability of an enterprise data metamodel, intermediate for all the involved information systems, simplifies this problem significantly [11].

We propose to apply this approach for solving the data integration task for an oil and gas enterprise due to its obvious advantages. Using an application domain metamodel allows making data exchange between the information systems simpler and more transparent; this approach guarantees that all the integration transactions will be properly understood and interpreted [10].

The availability of such data metamodel significantly reduces the quantity of the required adapters and it allows enterprise 
applications and information systems connecting via a common data model instead of connecting with each other via "expensive" pair adapters (fig. 3).
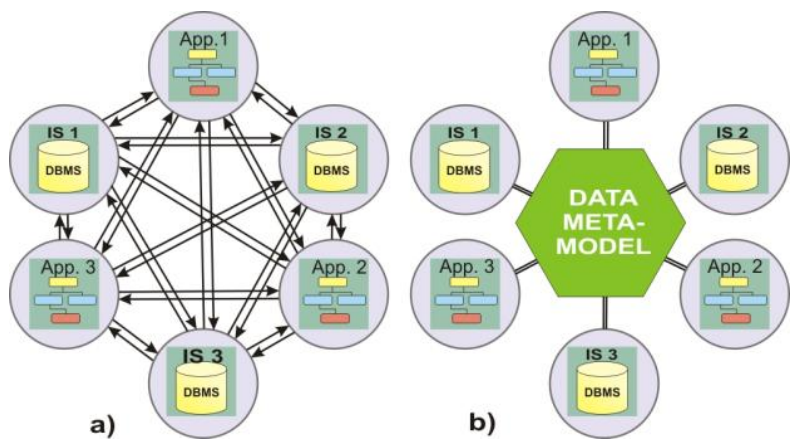

Fig. 3: Integration: «point-to-point» (a) and using the data metamodel (b)

The enterprise metamodel can be created on the basis of the conceptual models of information systems used within the enterprise. Actually, it is a creation of a new original data metamodel. Also, it is possible to apply the existing metamodel developed for the oil and gas branch.

Another way is less labor-consuming. There are a number of standards for the data integration for oil and gas application domain; we choose PRODML [12] as the most mature among them. We adopted this standard as a basis for a general oil and gas enterprise data metamodel.

\subsection{PRODML standard}

PRODML (Production XML) is the XML-standard developed by Energistics association in collaboration with the leading oil and gas and ICT companies: BP, Chevron, Shell, Statoil, Halliburton, Schlumberger, Microsoft Corporation and other organizations. It is aimed at supporting information interchange between applications and warehouses [12]. This standard is the extension of standard WITSML that is widely used for transferring information in the process of well drilling.

The main application areas of the standard are the following:

- Well fund information (list of wells; state of wells; well pressure, temperature, flow rate).

- Industrial equipment.

- Drilling and equipment.

- Staff.

- Surveys.

- Chemical analysis of production.

The primary goal which standard PRODML should solve consists in defining the data transfer standard between points $\mathrm{A}$ and $\mathrm{B}$; meanwhile, points $\mathrm{A}$ and $\mathrm{B}$ can be various oil companies, equipment and service suppliers, software, and, thus, the data can be of various time scales [13]. For example, it can be data of well survey, or real time data for on-line control of oil and gas production process.

The basis of PRODML is a hierarchical structure describing enterprise business objects (Product Flow Model). It consists of three basic elements: unit, network and model (fig. 4). The unit is any object for modeling or receiving information. It can represent both difficult objects (raw materials processing factory or a separator), and simple ones (valve or pump). Each unit contains ports, which allow connecting units among themselves by means of nodes. It is possible to connect modules "many to many". The network is a collection of connected modules. And, finally, the model is the representation of an enterprise which consists of networks set. The Product Flow Model hierarchy also defines the production movements between business objects of the enterprise and their directions (fig. 4).

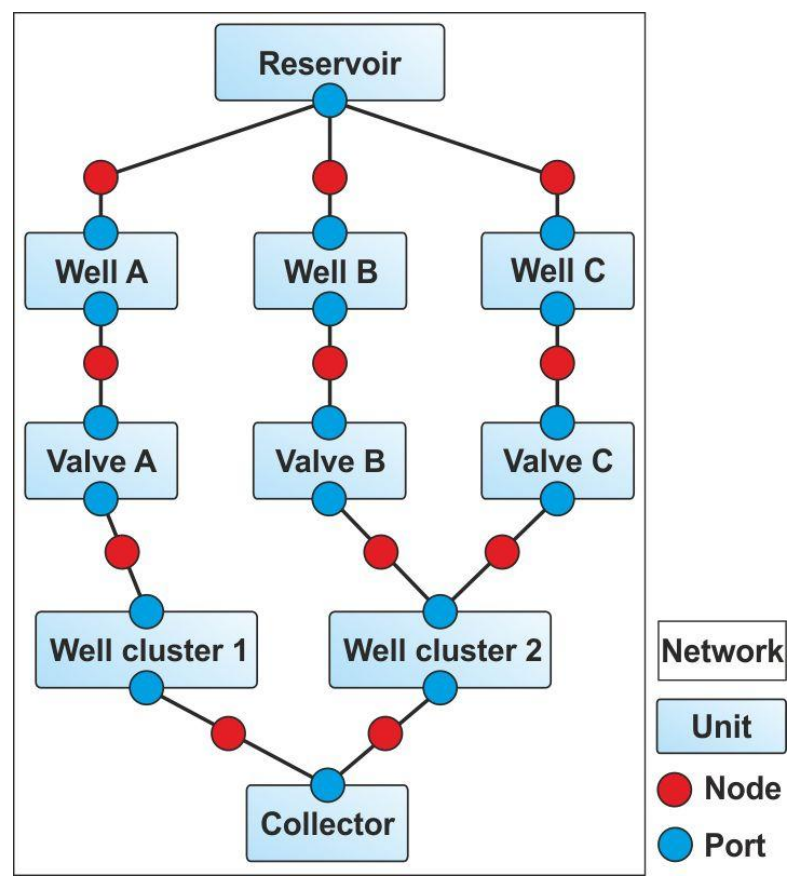

Fig. 4: Product Flow Model example [12]

Units and production flow characteristics are reflected in Product Volume Report. It is used for transmitting such information as the daily oil or gas production rate, or such characteristics as buffer, linear and annular pressure, temperature, flow rate, etc. The report can be both simple (one well data), and difficult (the whole field data).

The data model offered by standard PRODML covers the whole process from the moment of producing oil and gas till the moment of its realization. Using this data model makes it possible to significantly simplify integration task solving, but the standard model cannot cover all the features of an enterprise, each of which is unique and has its own peculiarities. Therefore, one needs tools allowing expanding PRODML for a concrete enterprise. At present, the adoption of standard PRODML is not supported by tools [13]. Therefore, the problem of the tools and implementation technology developing is crucial.

The data model presented in this work defines the ontology that allows describing the company business objects on its Information Technology (IT) platform. This will allow making the interpretation of all the elements in the company IT platform in a coherent way, establishing a common ontology to be used for integrating different available systems and applications.

\section{THE INTEGRATION PLATFORM ARCHITECTURE}

The main idea of the proposed platform is to combine integration approach based on domain model with SOA principles and to use standard PRODML as a core for the metamodel. The proposed integration platform architecture for the oil and gas enterprises is shown on fig. 5 . 


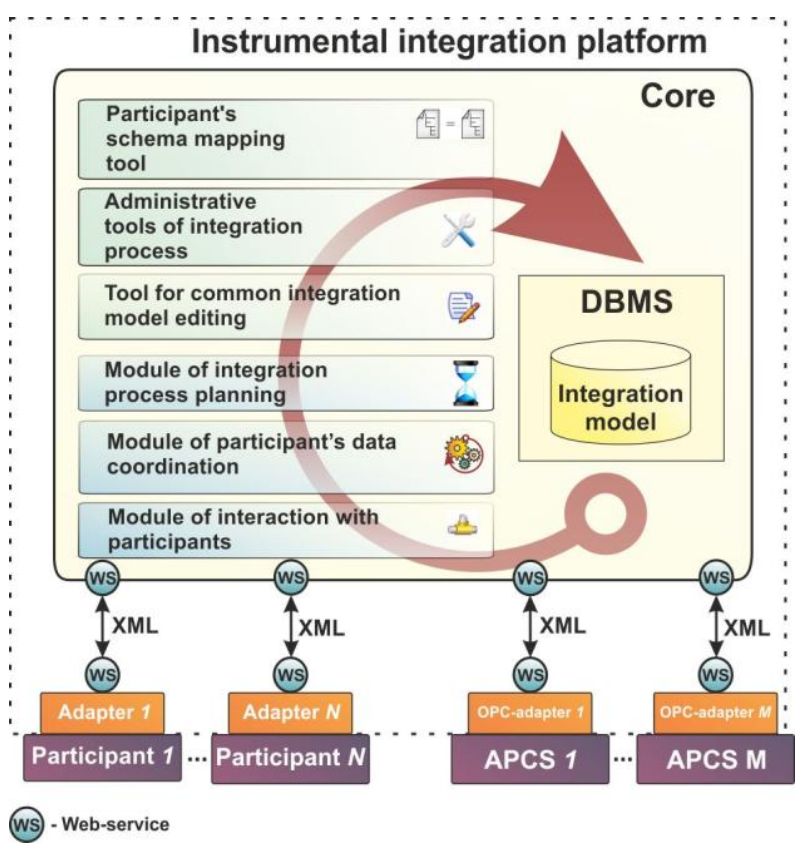

Fig. 5: Metamodel-based integration solution architecture for oil and gas enterprise

The data metamodel allows describing all the domain entities and relationship between them. It reflects a potentially possible structure of the enterprise ranging from technological data collection to the financial reporting and planning. For example: well fund, well surveys, drilling, repair work, etc.

The model based on the metamodel can be developed for a particular enterprise. This model (private model of an enterprise) reflects the structure of the enterprise objects, which are necessary to be involved in the process of data integration. Also, the model is supplemented with new objects if their involvement in the integration process is required. The private model solves the task objects data matching among various information systems of the enterprise.

The integration platform is a special application providing the interaction of all the participants involved, including APCS. It is not a place for detail data storage. The integration platform is the intermediary allowing organizing integration processes within the enterprise. The integration platform performs the following functions:

- It's a place for enterprise private model storage, it allows modifying it.

- It's a tool for creating, adjusting, performing and controlling all the integration operations.

- It's a tool for creation the rules for data transformation while transferring to the participants.

- It defines a data exchange mode (scheduled, on user's demand, on data change).

For each participant involved in an integration process it is necessary to have the adapter providing the integration platform with an interface to the participant data in the form, described by the enterprise metamodel. Creating the adapter for software connection to the integration platform is rather a labor-consuming task, because the adapter should transform the information from the integrated information system scheme to the scheme of metamodel, and this process cannot be formalized strictly. The adapter can represent both a service and an application with the user interface if there is a necessity to customize the process by user. Data exchange between adapters and an integration platform can be organized by means of Web-services in accordance with SOA architecture. It will provide flexibility and transparency of the whole system organization.

The architecture described above is taken as a principle for the tools developed and they help to put the suggested technology into practice:

- $\quad$ Product Flow Model Builder - the tool for the enterprise private model construction in accordance with standard PRODML (fig. 6);

- $\quad$ The adapter template for the information systems is allowing accelerating the creation process and allowing developer to avoid implementation of PRODML standard and the transport logic;

- Integration platform as the application realizing the functions described above.

\subsection{SOA}

An important aspect is the way of implementing the transportation layer of the information exchange between the integrated systems. Service Oriented Architecture (SOA) is a conventional approach to integration [4]. SOA is an approach for the software systems architecture organization offering to link the distributed systems functional units like services (web-services) which can be called by any requester program. SOA and web-services use a cross-platform interface between applications. The open standards describing web-services allow applying SOA for the interaction with all the information systems and applications used by the enterprise. Web-services are based on widespread and open protocols: HTTP, XML, UDDI, WSDL and SOAP. Web-services and SOA become popular and useful tools for enhancing integration solutions in order to improve business processes in the oil and gas industry. In recent years Enterprise Application Integration (EAI) systems have evolved towards SOA. That is why we suggest using SOA technology for the interaction of integrated information systems and applications of oil and gas enterprises.

\section{CASE STUDY}

Testing and approval of the developed platform carried out by means of its implementation in JSC «Tomskgazprom», which has modern oil and gas crafts. JSC «Tomskgazprom» provides a complete production cycle including drilling, production, preparation, processing, transportation and marketing of commercial products (stabilized gas condensate, gas and oil). Currently, the enterprise uses a number of different information systems: ERP system Microsoft Business Solution Axapta 3.0, MES "Magistral-Vostok," developed by Tomsk Polytechnic University, electronic document management system «OPTiMA WorkFlow» etc. Also it has a large number of specialized information systems for production purposes: «BASPRO» for geological models design [16] and «OISPipe» for intrafield pipe systems modeling [17]. There is a BI system based on the products of IBM Cognos for business analyses. Various APCS are used in the fields: they provide control of the technological processes on the wells, oil and gas pipelines, facilities for the preparation of gas and gas condensate, the booster compressor stations, etc. The main control systems are ROC, RS3, DeltaV, Siemens Simatic, etc.

The fragment of the integration platform scheme created for JSC "Tomskgazprom" is shown in Fig. 6. By means of the platform tools an integration model of the enterprise production data was built (wells attributes, wells hydrodynamic measurements results, information from technological facilities, etc.). 


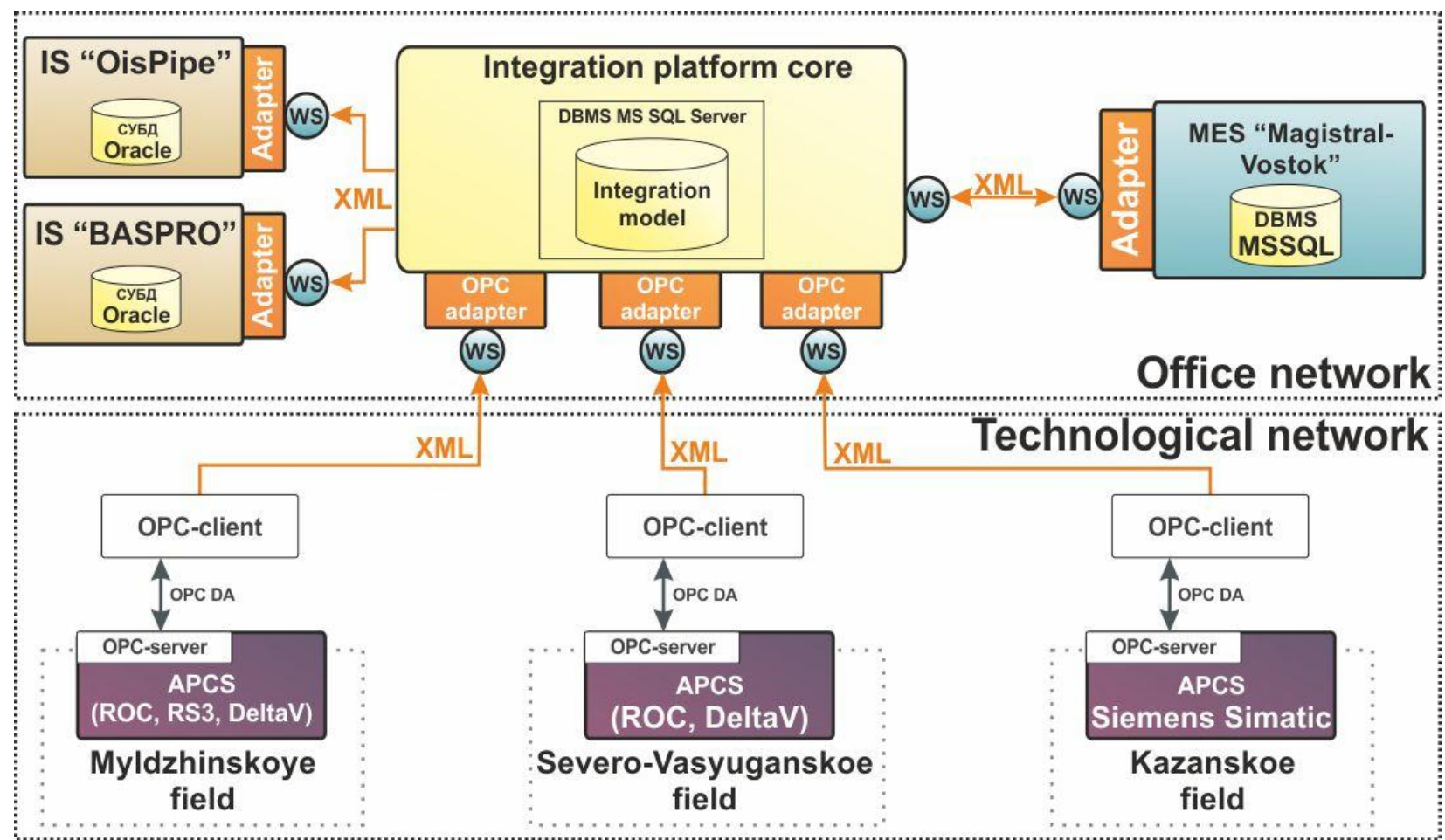

Fig. 6: Integration platform use case

An adapter was developed for each participant, which allows connecting to the core of the platform. All enterprise APCS are connected to the platform by means of OPC adapters. Matching of objects and their participants attributes are defined in the module of the integration processes configuration (fig. 7).

As a result, with the help of the implemented platform, field industrial objects data and wells fund objects data transmission was realized between these systems. MES «Magistral-Vostok» [15] with two specialized information systems: «BASPRO» and «OISPipe» [17] were used for "horizontal" integration. The same MES was integrated "vertically" by technological data with a number of APCS (Delta-V, RS/3, etc.) (fig. 6). The main transferred parameters are as follows:

- for wells - pressure (buffer, annular), temperature (linear, wellhead), well state, well maintenance way, action/dead time, well watering, raw materials component structure;

- for reservoir factor - water density, oil density, gas content;

- for well-stratum - raw materials extraction/release, water extraction.

\begin{tabular}{|c|c|c|}
\hline $\begin{array}{l}\text { Data from IS1 } \\
\text { (publisher) }\end{array}$ & Private enterprise model & $\begin{array}{c}\text { Data to IS2 } \\
\text { (subscriber) }\end{array}$ \\
\hline •Well 1, & $\begin{array}{l}\text {-Well fund } \\
\text { - Deposit } 1 \\
\text { - Well cluster } 1\end{array}$ & $\rightarrow$ Well 1 \\
\hline 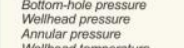 & 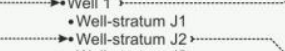 & 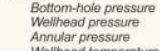 \\
\hline 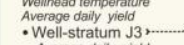 & 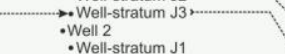 & 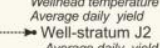 \\
\hline 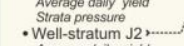 & 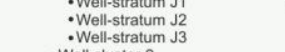 & 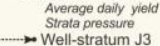 \\
\hline $\begin{array}{l}\text { Averegag daliy ryeld } \\
\text { Strata pressure }\end{array}$ & $\begin{array}{l}\text {-Well cluster } 2 \\
\text { - Well } 1 \text { - } \\
\text { : Wilstratum J2 } \\
\text {-Well-stratum J3 }\end{array}$ & $\begin{array}{l}\text { ANevage dailin yield } \\
\text { Strata pressure }\end{array}$ \\
\hline
\end{tabular}

Fig. 7: Integration objects correspondences customization on the basis of integration model
The implemented solution allowed simplifying databases populating of "BASPRO" and «OISPipe» systems with historical technological data, and also to provide these systems with the new data when coming from MES "Magistral-Vostok" avoiding repeated user's manual input

The integration of these systems provides a monthly transfer of about 880 thousand values of the various production parameters.

As a result of the integration all the systems were included in a common information space of JSC "Tomskgazprom." The platform has been approved as a reliable and flexible tool for the integration of enterprise production data.

\section{CONCLUSION}

This work concerns the problem of information systems integration by data. As a result we offered technologies for the minimization of expenses needed to solve this problem for oil and gas enterprises. The advantage of the considered technology is that it does not require reorganization of the enterprise IT structure and it could be implemented step-bystep when specific integration tasks appear. It significantly accelerates and simplifies the integration process, and also, it provides successful information system evolution within the enterprise information space development. Researches were provided under the support of Russian Federation Government within the state task "Science".

\section{REFERENCES}

[1] ISO. Industrial automation systems and integration Open systems application integration framework - Part 1: Generic reference description. Access mode: http://www.iso.org/iso/iso_catalogue/catalogue_tc/catalo gue_detail.htm?csnumber=30418.

[2] Microsoft BizTalk Server. Access mode: http://www.microsoft.com/biztalk/en/us/default.aspx. 
[3] WebSphere software. Access mode: http://www01.ibm.com/software/websphere.

[4] Juric M. 2007. SOA approach to integration. Packt Publishing Ltd., Birmingham.

[5] N. Markov, A. Saraykin, "Forming of the oil and gas company common information space," in Oil And Gas Journal. Russia, vol. 3, 2008, 34-41.

[6] What is OPC? Access mode: http://www.opcfoundation.org/Default.aspx/01_about/01 _whatis.asp?MID=AboutOPC.

[7] ECLIPSE 2010 - Reservoir Engineering Software. Access mode: www.slb.com/services/software/reseng/eclipse2010.aspx.

[8] IPM Products. Access mode: http://www.petex.com/products.

[9] Matrikon releases ProcessNet v3.1 for web-based decision support. Access mode: http://www.matrikon.com/news/56/index.aspx.

[10] Hollander D., High M., "Common Models in SOA: Tackling the Data Integration Problem". Access mode: www.progress.com/progress/dataxtend/docs/wp_do_not_ forget_data.pdf.

[11] Bravo C., Aguilar J., Ríos-Bolívar A., Aguilar-Martin J., Rivas-Echeverría F. A, "Generalized Data Meta-Model for Production Companies Ontology Definition", in International journal of system applications, engineering \& development, issue 4, vol. 2, 2008.

[12] PRODML. Reference Architecture PRODML 1.0 Access mode: http://www.prodml.org.

[13] Blaker J. PRODML scope statement version 2.0. Access mode: http://www.prodml.org.

[14] Cong Zhang, Abdollah Orangi, Amol Bakshi, Will Da Sie, and Viktor K. Prasanna. 2006. A service-oriented data composition architecture for integrated asset management. In Proceedings of SPE Intelligent Energy Conference and Exhibition (IECE), April 2006. Access mode: http://pgroup.usc.edu/iam/papers/SPE-99983DataComposition.pdf.

[15] S. Bogdan, A. Kudinov, N. Markov. 2009. Example of implementation of MES "Magistral-Vostok" for oil and gas production enterprise. In proceeding of Software Engineering Conference in Russia (CEE-SECR), 2009 5th Central and Eastern European, 131-136.

[16] BASPRO Optima. Access mode: http://www.baspro.ru/programm.

[17] OISPipe. Access mode: http://www.ois.ru/product.php?productName=OISPipe. 